\title{
Cinacalcet In the Treatment of Primary Hyperparathyroidism
}

Ifigenia Kostoglou-Athanassiou, Eleni Xanthakou, Panagiotis Athanassiou, Anastasios Gkountouvas, Philippos Kaldrymides

Department of Endocrinology, Red Cross Hospital, Athens, Greece

Endocrinologis, Sparti, Greece

Department of Rheumatology, St. Paul's Hospital, Thessaloniki, Greece

Endocrinologist, Pireaus, Greece

Department of Endocrinology, Metaxa Hospital, Pireaus, Greece 


\section{Introduction}

- Primary hyperparathyroidism is currently recognized with increasing frequency by routine calcium measurement in biochemical examinations

- Primary hyperparathyroidism may be due to a parathyroid adenoma, parathyroid hyperplasia and, rarely, parathyroid carcinoma

- Cinacalcet is used in the medical management of primary hyperparathyroidism 


\section{Aim}

- The aim was to assess the role of cinacalcet in the treatment of primary hyperparathyroidism 


\section{Methods}

- Patients with primary hyperparathyroidism $(n=20)$ (aged 5685 years) were studied

- Amongst them 4 patients had parathyroid hyperplasia and 16 had a parathyroid adenoma

- Calcium and PTH levels were increased in all patients

- All patients had ultrasonography and a 99mTc-Sestamibi scan

- In 16 patients a parathyroid adenoma was observed either on ultrasound or on scanning or in both

- In 4 of the patients a parathyroid adenoma was not localized by imaging 


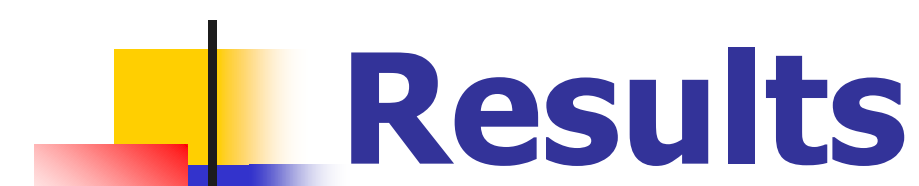

- Cinacalcet was used in all 16 parathyroid adenoma patients to normalize serum calcium levels prior to surgery

- In $\mathbf{1 0}$ of the parathyroid adenoma patients the adenoma was surgically excised, in a female aged 56, hyperparathyroidism recurring a year after surgery

- Sequentially, cinacalcet was administered at a dose of $\mathbf{3 0}$ mg twice daily and serum calcium levels normalized

- Within the group of patients with a parathyroid adenoma 6 were elderly, aged $>75$ years, with comorbidity and cinacalcet was administered at a dose of $\mathbf{3 0} \mathbf{~ m g}$ twice daily in $\mathbf{3}$ and $\mathbf{6 0}$ mg twice daily in 1 to avoid surgery

- In the group of patients with parathyroid hyperplasia cinacalcet was used for the treatment of hypercalcemia

- Within the whole group, 2 patients experienced mild gastrointestinal symptoms, but discontinuation of the drug could be avoided 


\section{Conclusions}

- Cinacalcet may be used for the treatment of primary hyperparathyroidism

- It can be used for the normalization of serum calcium prior to surgery, if surgery is not an option, in the event of recurrence after surgery and in parathyroid hyperplasia 